\title{
THE EMERGING ROLE OF SERRATIOPEPTIDASE IN ORAL SURGERY: LITERATURE UPDATE
}

\author{
SANTHOSH KUMAR MP* \\ Department of Oral and Maxillofacial Surgery, Saveetha Dental College and Hospital, Saveetha Universty, Velappanchavadi, \\ Chennai -600 077, Tamil Nadu, India. Email: santhoshsurgeon@gmail.com
}

Received: 03 November 2017, Revised and Accepted: 07 December 2017

\begin{abstract}
Serratiopeptidase, a proteolytic enzyme derived from Serratia E-15 species enterobacteria, is widely used in medical field for its anti-inflammatory, anti-edemic properties, and analgesic properties. It is being used commonly in various specialties such as orthopedics, otolaryngology, gynecology, surgery, pulmonology, ophthalmology, and dentistry. Research has shown that serratiopeptidase is the most effective anti-inflammatory agent compared to other enzyme preparations. This article reviews the efficacy, safety, and applications of serratiopeptidase in oral surgery. This article also discusses the mechanism of action of serratiopeptidase, its contraindications and complications. From the recently published literature, it is clear that the role of serratiopeptidase as a therapeutic agent in oral and maxillofacial surgery is expanding and they hold a promising future as a broad-spectrum anti-inflammatory drug with minimal side effects and complications. Further, research will broaden their applications in the field of medicine and dentistry.
\end{abstract}

Keywords: Analgesic, Anti-inflammatory, Oral surgery, Infections, Proteolytic enzymes, Serrapeptase, Serratiopeptidase, Third molar surgery.

(C) 2018 The Authors. Published by Innovare Academic Sciences Pvt Ltd. This is an open access article under the CC BY license (http://creativecommons. org/licenses/by/4. 0/) DOI: http://dx.doi.org/10.22159/ajpcr.2018.v11i3.23471

\section{INTRODUCTION}

Enzymes are extremely potent substances and their therapeutic applications in medical field are increasing due to its efficacy and safety. Enzymes are derived from plants (papase and bromelain), animals (trypsin and chymotrypsin), bacteria (streptokinase, streptodornase, and serratiopeptidase), and fungi [1].

Serratiopeptidase (serralysin/serrapeptase/Serratia-protease) is a proteolytic enzyme which is predominantly used for its anti-inflammatory properties. Initially, trypsin, chymotrypsin, and bromelain were used [2]. During the 1950s in the USA, parenteral trypsin was found to be very useful in treating inflammation associated with sports injuries, rheumatoid arthritis, ulcerative colitis, atypical viral pneumonia, and postsurgical edema. Serratiopeptidase was first used in Japan for therapeutic purposes [3]. Later parenteral enzymes were replaced by enteric-coated oral formulations. Research conducted in Japan and Europe during the 1980s and 1990s showed that serratiopeptidase was the most effective anti-inflammatory agent compared to other enzyme preparation $[4,5]$. Since then it is in use for over 40 yrs in Europe and Japan for the treatment of pain and inflammation associated with various conditions. Recent literature studies reported that serratiopeptidase can be used as an effective anti-inflammatory agent after oral surgical procedures. This article reviews the properties, mechanism of action, efficacy, and uses of serratiopeptidase in oral surgery and their future applications.

\section{SYNTHESIS OF SERRATIOPEPTIDASE}

Serratiopeptidase is produced by Serratia species E-15, non-pathogenic enterobacteria, found naturally in silkworm Bombyx mori which helps the emerging moth in dissolving its cocoon. Serratiopeptidase for medical purposes is obtained by purification from culture of Serratia E-15 species bacteria (fermentation biotechnology) [6].

\section{CHEMICAL STRUCTURE}

Serratiopeptidase is a metalloprotease containing a zinc atom with a molecular weight of 45,000-60,000 and consists of 470 amino acids which are important for its proteolytic activity (Fig. 1). The enzyme was characterized to be free of any sulfur-containing amino acids such as cysteine and methionine [7,8]. The enzyme belongs to serralysin group of enzymes EC number 3.4.24.40 and is known to cleave the peptides with linkages of Asn-Gln, CysSO3H-Gly, Arg-Gly, and Tyr-tyr as well as the bond between His-Leu, Gly-Ala, Ala-Leu, Tyr-leu, Gly-Gly, Phen-Tyr, and Tyr-Thr, showing broad substrate specificity. The enzyme has maximal activity at $\mathrm{pH} 9.0$ and at a temperature of $40^{\circ} \mathrm{C}$ and is completely inactivated by heating at $55^{\circ} \mathrm{C}$ for $15 \mathrm{~min}$ [9].

\section{ADMINISTRATION OF SERRATIOPEPTIDASE}

It is preferred over other proteolytic enzymes such as trypsin and chymotrypsin as it has high potency with negligible side effects. It is administered orally as an enteric-coated tablet to avoid degradation by enzymes in gastrointestinal tract. Normal dosage is $10 \mathrm{mg}$ [20,000 U] 3 times a day to be taken on an empty stomach or $2 \mathrm{~h}$ after food. It is available as a sole drug or in combination with other enzyme preparations and analgesics. It is usually available as a fixed dose combination with non-steroidal anti-inflammatory drugs (NSAIDs) such as diclofenac, aceclofenac, ibuprofen, tramadol, and paracetamol. In the treatment of infections, it is administered with various antimicrobials as it increases their tissue penetration. It is orally effective and equivalent to diclofenac sodium as an anti-inflammatory agent in both acute and chronic phases of inflammation [10].

Kaur and Singh [11] designed and developed albumin nanoparticles that can be used to effectively deliver serratiopeptidase at the site of inflammation in treating conditions like rheumatoid arthritis. For children, powder form of enzymes is also available. It is advised not to take serratiopeptidase for more than 4 weeks for chronic inflammatory or infective conditions. This can prevent clot formation and can cause serious bleeding problems for those with bleeding disorders or in the event of an injury; hence, it has to be stopped at least 2 weeks before any surgical procedure. Administered orally it is absorbed from intestine, enters into systemic circulation in unchanged form and can penetrate into all tissues through blood or lymph, especially inflamed areas in an enzymatically active form [12]. Serratiopeptidase binds to alpha-2macroglobulin in the blood in a 1:1 ratio, thus does not elicit allergic reaction but retains its enzymatic activity and high concentration of drug is transferred to the site of infection/inflammation in 1 hour $[13,14]$. 


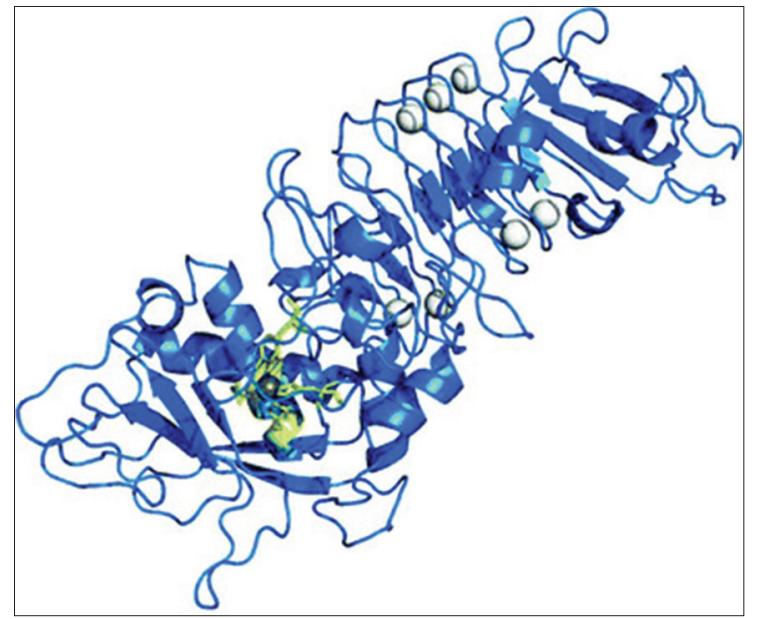

Fig. 1: Chemical structure of serratiopeptidase

\section{MECHANISM OF ACTION}

Yamasaki et al., [3] in 1967, showed that serratiopeptidase is a potential anti-inflammatory agent. It decreases the amount of fluid in the tissues, thins the fluids, and facilitates the drainage of the fluid, thereby reduces swelling. This also enhances tissue repair and reduces pain. It dissolves the dead tissue surrounding the injured area without harming living tissue, thereby accelerating healing. Several studies have proved that orally administered proteolytic enzymes can specifically control the inflammatory cytokines and the onset of chronic inflammation [15-17]. Serrapeptidase and other proteolytic enzymes are effective in controlling and modulating inflammatory processes are referred to as "adjunct therapeutic agents" [18]. It also modifies cell-surface adhesion molecule that guides inflammatory cells to their target site of inflammation. These adhesion molecules are known to play an important role in the development of arthritis and other autoimmune disorders [19].

Serratiopeptidase alleviates pain by inhibiting the release of bradykinin from inflamed tissues. It also acts by breaking down fibrin and other dead or damaged tissue, thus dissolving blood clots and atherosclerotic plaques.

Serratiopeptidase hydrolyses bradykinin, histamine, and serotonin thereby reduces swelling and improves microcirculation and expectoration of sputum [20]. Thus, it reduces capillary permeability and also breakdown of proteins and exudates, and hence supports wound healing. It decreases neutrophil count and alters the viscoelasticity of sputum, thereby clears mucous secretions in patients with chronic airway disease $[4,21]$.

Selan et al. [22] from their research identified that serratiopeptidase can impair the biofilm formation by Staphylococcus aureus on prostheses, catheters, and medical devices. Thus, it has a potential antipathogenic action apart from its proteolytic activity. Serratiopeptidase modulates adhesion expression in some bacterial species and enhances antibiotic efficacy toward biofilm-forming bacteria [23-25]. The antibiofilm formation property of serratiopeptidase helps in enhancing antibiotic efficacy in the treatment of staphylococcal infections [26].

While anti-inflammatory drugs [NSAIDs and corticosteroids] may offer temporary, symptomatic relief from pain, swelling, and inflammation, they may also be immunosuppressive and are known to have gastrointestinal side effects. Serratiopeptidase reduces pain and swelling without inhibiting prostaglandins and has no gastrointestinal adverse effects. It also acts by enhancing blood circulation due to proteolytic effect, removing damaged and denatured proteins and cellular debris, and modulating inflammatory cytokines. Thus, serratiopeptidase has anti-inflammatory, analgesic, anti-edemic, antipathogenic, fibrinolytic and caseinolytic properties. In contradiction, Joshi and Nerurkar et al. [27] conducted a study on rats (paw model) to evaluate the efficacy of serratiopeptidase in reducing edema. They concluded that serratiopeptidase was not an effective anti-inflammatory agent either alone or with diclofenac and further studies had to be done in humans.

\section{CONTRA-INDICATIONS}

It is contraindicated in pregnancy, lactation, hemophiliacs, liver damage, renal dysfunction and in patients taking antiplatelet drugs (aspirin and clopidogrel), anticoagulants (warfarin), garlic, fish oil, and turmeric as it can cause an increased risk of bleeding or bruising.

\section{COMPLICATIONS}

They are very safe drugs. Allergy or anaphylaxis to enzymes is very rare. Adverse drug reactions are reported very rarely which includes Stevens-Johnson syndrome, erythema multiforme, anorexia, nausea, diarrhea, vomiting, epistaxis, hemoptysis, pneumonitis, and coagulation abnormalities. High doses of serratiopeptidase could cause esophageal ulcers, acute eosinophilic pneumonia, subepidermal bullous dermatosis, and inability to move. Saibal Moitra et al. [28] reported a case in which a patient developed Stevens-Johnson syndrome after ingestion of diclofenac-serratiopeptidase drug combination. Rajaram et al. [29] reported a case of buccal space abscess treated with serratiopeptidase which lead to the spread of infection to the deeper tissue planes. Hence, serratiopeptidase has to be used with caution in treating abscess cases.

\section{CLINICAL APPLICATIONS OF SERRATIOPEPTIDASE}

\section{General uses}

It is used in many branches of medicine such as surgery, orthopedics, pulmonology, gynecology, dentistry, otorhinolaryngology, and ophthalmology [30].

It is indicated for the conditions such as back pain, fibromyalgia, migraine headache, rheumatoid arthritis, tension headache, osteoporosis, osteoarthritis, carpal tunnel syndrome, laryngitis, sinusitis, pharyngitis, catarrhal rhinopharyngitis, tonsillitis, chronic bronchitis, bronchial asthma, chronic obstructive pulmonary disease, Crohn's disease, ear infections, ulcerative colitis, heart disease, leg ulcers, autoimmune diseases, psoriasis, allergies, keratitis, uveitis, endophthalmitis, subconjunctival hemorrhage, hyphemas, vitreous hemorrhage, infections, diabetes, atherosclerosis, facial swelling, fibrocystic breast disease, cystitis, epididymitis, episiotomy, hysterectomy, thrombophlebitis, hematomas, septic abortion, pelvic inflammatory disease, salpingitis, sprains and torn ligaments, lacerations, and other traumatic injuries, edema, as well as postoperative inflammation $[31,32]$.

It is taken as a health supplement for many conditions to prevent morbidity (e.g., cardiovascular morbidity). German researchers have shown that serrapeptase helps to digest atherosclerotic plaque without harming healthy cells lining the arterial wall; thus, it can be used to treat atherosclerosis [33].

\section{Anti-inflammatory action}

In the study by Esch et al. [34], serratiopeptidase produced good pain relief and 50\% reduction in swelling when administered for knee ligament surgery. In a double-blind study, serratiopeptidase was superior to placebo for the improvement of breast pain, swelling, and induration [35]. The efficacy and tolerability of serratiopeptidase were evaluated in a multicenter, double-blind, and placebo-controlled study of 193 subjects suffering from acute or chronic ear, nose, or throat disorders, and it was found that serratiopeptidase was very effective in reducing localized inflammation and tolerance was found to be very good [4]. In a rat model study by Mundhava et al. [36], it was shown that proteolytic enzymes such as serratiopeptidase, trypsin, and chymotrypsin have significant anti-inflammatory activity either alone or in combination with diclofenac. These enzymes do not possess analgesic action, but they potentiate the peripheral analgesic action of 
diclofenac at therapeutic equivalent doses. According to Viswanatha Swamy and Patil [37], proteolytic enzymes have anti-inflammatory activity and exhibit synergistic effects with aspirin in rat inflammation models in both acute and subacute phases of inflammation. A study showed that serratiopeptidase can be used in the conservative treatment of patients with carpal tunnel syndrome [38]

\section{THERAPEUTIC APPLICATIONS IN ORAL SURGERY}

Serratiopeptidase is used mainly in post-traumatic and post-operative inflammatory conditions (after any minor and major oral surgical procedures), orodental infections.

\section{Third molar surgery}

Surgical removal of impacted lower third molar teeth is a very common procedure in oral surgery. It is always associated post-operative swelling, pain, and trismus. Various studies were conducted to evaluate an effective drug to control the post-operative complications. Enzyme serrapeptase was found to be very useful in controlling post-operative swelling.

Al-Khateeb and Nusair [39] conducted a prospective, intraindividual, randomized, double-blinded, and crossover study on 24 patients. Treatment group received $5 \mathrm{mg}$ serratiopeptidase with $1000 \mathrm{mg}$ paracetamol tablets and control group was given placebo with $1000 \mathrm{mg}$ paracetamol for 7 days. Serratiopeptidase caused significant reduction in swelling and pain intensity in the $2^{\text {nd }}, 3^{\text {rd }}$, and $7^{\text {th }}$ post-operative day after lower third molar surgery. However, the drug had no effect on post-operative trismus.

Murugesan et al. [40] carried out a randomized study on 110 patients to evaluate the usefulness of serratiopeptidase compared to dexamethasone in impacted lower third molar surgery. Postoperatively, $1 \mathrm{mg}$ dexamethasone was given thrice daily in the first group and $10 \mathrm{mg}$ serratiopeptidase was given thrice daily in the second group for 3 days. The patients were reviewed on the $1^{\text {st }}, 3^{\text {rd }}, 5^{\text {th }}$, and $7^{\text {th }}$ postoperative days. They found that dexamethasone was more effective in reduction of post-operative swelling and pain when compared to serratiopeptidase. Hence, they concluded serratiopeptidase can be used as an alternative to corticosteroids to control inflammation in cases where corticosteroids are contraindicated. Both the drugs had no beneficial effects in relieving trismus.

Chappi et al. [41] conducted a similar study on 100 patients to find the efficacy of serratiopeptidase in the management of post-operative complications after lower third molar surgery. The patients were randomly divided into two groups and drugs were administered orally in divided doses. Postoperatively, one group received methylprednisolone $12 \mathrm{mg} /$ day and the other group was given serratiopeptidase $20 \mathrm{mg} /$ day for 5 days. The patients were followed-up on the $1^{\text {st }}, 3^{\text {rd }}$, and $5^{\text {th }}$ post-operative days. On comparing methylprednisolone with serratiopeptidase, they concluded that methylprednisolone exhibited better pain relief, while serratiopeptidase is very effective in controlling postsurgical swelling and trismus and did not affect the wound healing. Hence, synergistic combinations of these two drugs would be more effective than individual drug when widespread post-operative sequelae are expected after lower third molar surgery.

Al-Sandook et al. [42] did a study on 30 patients in which 15 patients were given serratiopeptidase and 15 patients received conventional treatment for 3 days. They demonstrated that proteolytic enzymes possessed good anti-inflammatory and analgesic properties when administered for lower third molar surgery.

Chopra et al. [43] evaluated and compared the efficacy and safety of paracetamol, serratiopeptidase, ibuprofen, and betamethasone in reducing swelling and pain following lower third molar surgery. In this randomized, double-blinded, and placebo-controlled trial, 150 patients were randomly divided into one of the five groups to receive treatment 3 times a day for 7 days with ibuprofen $600 \mathrm{mg}$, betamethasone
$0.5 \mathrm{mg}$, paracetamol $1 \mathrm{~g}$, serratiopeptidase $20 \mathrm{mg}$, or placebo. Serratiopeptidase showed analgesic and anti-inflammatory activity, but it was not significant, whereas ibuprofen and betamethasone were effective in reducing post-operative swelling and pain compared to serratiopeptidase or paracetamol groups.

Chaiwat et al., [44] in their study to evaluate the efficacy of serratiopeptidase on post-operative pain, swelling, and trismus following lower third molar surgery, found no significant difference between the serratiopeptidase and control groups. In the study by Surachai [45], no significant difference was found between the serratiopeptidase group and nil medication group in the reduction of facial swelling after impacted lower third molar surgery. It was a trial with small sample size of 40 patients which is a limitation of the study.

\section{Infections}

It helps in prevention and treatment of a variety of infections. Serratiopeptidase enhances the bactericidal effect of antibiotics in cultures and prevents the formation of biofilms, hence useful in treating prosthetic infections [22]. It increases the permeability of desired tissues which enhances the absorption of antibiotics to the site of infections, thereby potentiating the action of antibiotics $[46,47]$. Thus, they are a useful adjunct to antibiotics in both acute and chronic infections. Selan et al. [48] in their in vitro study showed that serratiopeptidase greatly enhances the activity of antibiotics (ofloxacin) on sessile cultures of pathogens and can inhibit biofilm formation. Thus, serratiopeptidase can be used for the treatment of prosthetic device infections and infections caused by biofilm-forming bacteria. Serratiopeptidase enhances the activities of many antibiotics by improving the penetration of antibiotics in infected sites.

It is useful in orodental infections and reduces the healing time by $50 \%$ [49]. Intra-articular serratiopeptidase has also been found useful in the eradication of infection caused by biofilm-forming bacteria in experimental animal model. It is very useful in controlling airway infections and inflammations. In many studies, it has been shown that using serratiopeptidase and/or other proteolytic enzymes along with antibiotics or other medications, healing occurs much faster $[50,51]$.

\section{Major surgical procedures}

Tachibana et al. [52] conducted a multicenter, randomized, and double-blinded study to evaluate the efficacy of serratiopeptidase in 174 patients undergoing Caldwell-Luc antrotomy for chronic empyema. 88 patients in the treatment group received $30 \mathrm{mg} /$ day of serratiopeptidase and 86 patients in the control group received placebo drugs. The degree of post-operative swelling in the serrapeptasetreated patients was significantly less than in the placebo group and no side effects were reported with the drug.

\section{Traumatic injuries}

Garg et al. [53] evaluated and compared the efficacy and safety of serratiopeptidase and aceclofenac in reducing swelling and pain following traumatic soft tissue injury. Serratiopeptidase showed significant anti-inflammatory effect with mild analgesic action, whereas aceclofenac exhibited superior analgesic effect as compared to serratiopeptidase. Serratiopeptidase can be used as an antiinflammatory agent in traumatic bony and soft tissue injuries in oral and maxillofacial region.

\section{DISCUSSION}

Inflammation occurs due to many conditions in humans. NSAIDs and corticosteroids are the commonly used drugs to combat inflammation. However, they are not without side effects and are also costly; hence, their judicious use is mandatory. Serratiopeptidase can be a useful alternative to these drugs as an anti-inflammatory agent in conditions where the former drugs are contraindicated [54]. It can be prescribed alone or preferably in combination with other analgesics when superior analgesic action is desired. When prescribed in combination with NSAIDs/corticosteroids, serratiopeptidase has superior anti-inflammatory and analgesic action. 
Proteolytic enzymes can play an important role in future in treating patients with nosocomial, viral, and resistant infections, especially in pediatric and geriatric age groups. Its safety profile, lack of development of resistance, and inability to show tolerance makes it a preferred supplemental drug to many antimicrobials to treat infections $[55,56]$.

Serrapeptidase is produced in highly controlled laboratory conditions adhering to good clinical practice protocols. Serratiopeptidase alone or in combination with NSAIDs is costly and has been marketed widely by the pharmaceutical companies. It should be prescribed if the benefits of the drug outweigh the cost burden to the patient. Thus, prescription of proteolytic enzymes should be decided on evidence-based medicine and not to be influenced by medical representatives [57]. Although the clinical efficacy and safety of serratiopeptidase has been proven in many studies, most of the evidence is based on animal experiments, few uncontrolled clinical trials, and randomized controlled clinical trials of poor quality. Thus, there is a need for further extensive experimental and clinical research in this area [30,31,58-60].

\section{CONCLUSION}

Serratiopeptidase has a well proven excellent anti-inflammatory action with moderate analgesic properties. Serratiopeptidase can be used for effectively managing swelling and pain after any minor/major dental or oral surgical procedures and facial trauma either alone or in combination with NSAIDs/corticosteroids. It can be used as an adjunct therapeutic agent along with antimicrobials in the treatment of orofacial infections. Furthermore, its use and application in the areas of human health are well documented and continue to be a major aspect of inflammation and pain management protocol in many clinics around the world. The role of serratiopeptidase as a therapeutic agent in oral and maxillofacial surgery is expanding, and they hold a promising future as a broad-spectrum antiinflammatory drug with minimal side effects and complications.

\section{REFERENCES}

1. Sherry S, Fletcher AP. Proteolytic enzymes: A therapeutic evaluation. Clin Pharmacol Ther 1960;1:202-26.

2. Ambrus JL, Lassman HB, DeMarchi JJ. Absorption of exogenous and endogenous proteolytic enzymes. Clin Pharmacol Ther 1967;8:362-8.

3. Yamasaki H, Tsuji H, Saeki K. Anti-inflammatory action of a protease, TSP, produced by Serratia. Nihon Yakurigaku Zasshi 1967;63:302-14.

4. Mazzone A, Catalani M, Costanzo M, Drusian A, Mandoli A, Russo S, et al. Evaluation of Serratia peptidase in acute or chronic inflammation of otorhinolaryngology pathology: A multicentre, double-blind, randomized trial versus placebo. J Int Med Res 1990;18:379-88.

5. Kakinuma A, Moriya N, Kawahara K, Sugino H. Repression of fibrinolysis in scalded rats by administration of Serratia protease. Biochem Pharmacol 1982;31:2861-6.

6. Miyata K, Maejima K, Tomoda K, Isono M. Serratia protease Part I. Purification and general properties of the enzyme. Agric Biol Chem 1970;34:310-8.

7. Kaviyarasi NS, Prashantha CN, Suryanarayan VV. In silico analysis of inhibitor and substrate binding site of serrapeptidase from Serratia marcescens MTCC 8708. Int J Pharm Pharm Sci 2016;8:975-1491.

8. Indrayati A, Asyarie S, Suciati T, Retnoningrum DS. Study on the properties of purified recombinant superoxide dismutase from Staphylococcus equorum, a local isolate from Indonesia. Int J Pharm Pharm Sci. 2014;6(11):1-6.

9. Nakahama K, Yoshimura K, Marumoto R, Kikuchi M, Lee IS, Hase T, et al. Cloning and sequencing of Serratia protease gene. Nucleic Acids Res 1986;14:5843-55

10. Jadav SP, Patel NH, Shah TG, Gajera MV, Trivedi HR, Shah BK, et al. Comparison of anti-inflammatory activity of Serration peptidase and diclofenac in albino rats. J Pharmacol Pharmacother 2010;1:116-7.

11. Kaur H, Singh A. Design, development and characterization of Serration peptidase loaded albumin nanoparticles. J App Pharm Sci 2015;5:103-9.

12. Malshe PC. Orally administered Serration peptidase: Can it work? J Assoc Physicians India 1998;46:492.

13. Moriya N, Nakata M, Nakamura M, Takaoka M, Iwasa S, Kato K, et al. Intestinal absorption of serrapeptase (TSP) in rats. Biotechnol Appl Biochem 1994;20:101-8.

14. Moriya N, Shoichi A, Yoko H, Fumio H, Yoshiaki K. Intestinal absorption of serrapeptase and its distribution to the inflammation sites. Jpn Pharmacol Ther 2003;31:659-66.

15. Yamasaki H, Tsuji H, Saeki K. Anti-inflammatory action of a protease, TSP, produced by Serratia. Nihon Yakurigaku Zasshi 1967;63:302-14

16. Heumann D, Vischer TL. Immunomodulation by alpha 2-macroglobulinprotease complexes: The effect on the human T-lymphocyte response. Eur J Immunol 1988;18:755.

17. Desser L, Rehberger A. Induction of tumor necrosis factor in human peripheral-blood mononuclear cells by proteolytic enzymes. Oncology 1990;47:475-7.

18. Hale LP, Haynes BF. Bromelain treatment of human T-cells removes CD44, CD45RA, E2/MIC2, CD6, CD7, and Leu81/LAM 1 surface molecules and markedly enhances CD2 mediated T-cell activation. J Immunol 1992;149:3809.

19. LaMarre J, Wollenberg GK, Gonias SL, Hayes MA. Cytokine binding and clearance properties of proteinase-activated alpha 2-macroglobulins. Lab Invest 1991;65:3-14

20. Desser L, Rehberger A, Kokron E, Paukovits W. Cytokine synthesis in human peripheral blood mononuclear cells after oral administration of polyenzyme preparations. Oncology 1993;50:403-7.

21. Nakamura S, Hashimoto Y, Mikami M, Yamanaka E, Soma T, Hino M, et al. Effect of the proteolytic enzyme serrapeptase in patients with chronic airway disease. Respirology 2003;8:316-20.

22. Braga PC, Moretti M, Piacenza A, Montoli CC, Guffanti EE. Effects of seaprose on the rheology of bronchial mucus in patients with chronic bronchitis. A double-blind study vs placebo. Int J Clin Pharmacol Res 1993;13:179-85.

23. Selan L, Papa R, Tilotta M, Vrenna G, Carpentieri A, Amoresano A, et al. Serratio peptidase: A well-known metalloprotease with a new non-proteolytic activity against $S$. aureus biofilm. BMC Microbiol 2015;15:207

24. Longhi C, Scoarughi GL, Poggiali F, Cellini A, Carpentieri A, Seganti L, et al. Protease treatment affects both invasion ability and biofilm formation in Listeria monocytogenes. Microb Pathog 2008;45:45-52.

25. Artini M, Scoarughi GL, Papa R, Cellini A, Carpentieri A, Pucci P, et al. A new anti-infective strategy to reduce adhesion-mediated virulence in Staphylococcus aureus affecting surface proteins. Int J Immunopathol Pharmacol 2011:24:661-72.

26. Papa R, Artini M, Cellini A, Tilotta M, Galano E, Pucci P, et al. A new anti-infective strategy to reduce the spreading of antibiotic resistance by the action on adhesion-mediated virulence factors in Staphylococcus aureus. Microb Pathog 2013;63:44-53.

27. Mecikoglu M, Saygi B, Yildirim Y, Karadag-Saygi E, Ramadan SS, Esemenli T, et al. The effect of proteolytic enzyme Serration peptidase in the treatment of experimental implant-related infection. J Bone Joint Surg Am 2006;88:1208-14.

28. Joshi KK, Nerurkar RP. Anti-inflammatory effect of the Serration peptidase - rationale or fashionable: A study in rat paw oedema model induced by the carrageenan. Indian J Physiol Pharmacol 2012;56:367-74

29. Moitra S, Sen S, Banerjee I, Das P, Tripathi SK. Diclofenac- Serratio peptidase combination induced stevens - Johnson syndrome - A rare case report with review of literature. J Clin Diagn Res 2014;8:8-11.

30. Rajaram P, Bhattacharjee A, Ticku S. Serratio peptidase - A cause for spread of infection. J Clin Diagn Res 2016;10:ZD31-2.

31. Bhagat S, Agarwal M, Roy V. Serratio peptidase: A systematic review of the existing evidence. Int J Surg 2013;11:209-17.

32. Tiwari M. The role of Serratio peptidase in the resolution of inflammation. Asian J Pharm Sci 2017;12:209-15.

33. Sadanobu K, Shoji T, Nishimura Y, Miyazaki S. Effect of combination therapy with Danzen tablets and antibiotics for acute cystitis. Acta Urol Jpn 1967;13:853-7.

34. Cooney DA, Rosenbluth RJ. Enzymes as therapeutic agents. Adv Pharmacol Chemother 1975;12:185-289

35. Esch PM, Gerngross H, Fabian A. Reduction of postoperative swelling. Objective measurement of swelling of the upper ankle joint in treatment with serrapeptase-- a prospective study. Fortschr Med 1989;107:67-8,71-2.

36. Kee WH, Tan SL, Lee V, Salmon YM. The treatment of breast engorgement with serrapeptase (Danzen): A randomised double-blind controlled trial. Singapore Med J 1989;30:48-54.

37. Mundhava SG, Mehta DS, Thaker SJ. A comparative study to evaluate anti-inflammatory and analgesic activity of commonly used proteolytic enzymes and their combination with diclofenac in rats. Int J Pharm Sci Res 2016;7:2615.

38. Viswanatha Swamy AH, Patil PA. Effect of some clinically used 
proteolytic enzymes on inflammation in rats. Indian $\mathrm{J}$ Pharm Sci 2008;70:114-7.

39. Panagariya A, Sharma AK. A preliminary trial of Serration peptidase in patients with carpal tunnel syndrome. J Assoc Physicians India 1999;47:1170-2

40. Al-Khateeb TH, Nusair Y. Effect of the proteolytic enzyme serrapeptase on swelling, pain and trismus after surgical extraction of mandibular third molars. Int J Oral Maxillofac Surg 2008;37:264-8.

41. Murugesan K, Sreekumar K, Sabapathy B. Comparison of the roles of Serration peptidase and dexamethasone in the control of inflammation and trismus following impacted third molar surgery. Indian J Dent Res 2012;23:709-13.

42. Chappi DM, Suresh KV, Patil MR, Desai R, Tauro DP, Bharani KN, et al. Comparison of clinical efficacy of methylprednisolone and Serration peptidase for reduction of postoperative sequelae after lower third molar surgery. J Clin Exp Dent 2015;7:e197.

43. Al-Sandook TA, Tawfik NO, Qassim DA. Clinical evaluation of the efficacy of orthal-forte (prolytic enzymes, trypsin and chymotrypsin) on postoperative sequel following the removal of lower impacted third molar. Int J Enhanc Res Sci Technol Eng 2014;3:169-73.

44. Chopra D, Rehan HS, Mehra P, Kakkar AK. A randomized, doubleblind, placebo-controlled study comparing the efficacy and safety of paracetamol, Serration peptidase, ibuprofen and betamethasone using the dental impaction pain model. Int J Oral Maxillofac Surg 2009;38:350-5.

45. Chaiwat S, Suddhasthira T, Nuntasanti V. Effect of Serratio peptidase on postoperative swelling after removal of impacted mandibular third molar. Mahidol Dent J 1991;11:(e-journal).

46. Surachai. A Postoperative Study in Dental Patients After Extraction of $3^{\text {rd }}$ Molar; 1981

47. Okumura H, Watanabe R, Kotoura Y, Nakane Y, Tangiku O. Effects of a proteolytic-enzyme preparation used concomitantly with an antibiotic in osteoarticular infections (author's transl). Jpn J Antibiot 1977;30:223-7.

48. Koyama A, Mori J, Tokuda H, Waku M, Anno H, Katayama T, et al. Augmentation by serrapeptase of tissue permeation by cefotiam. Jpn J
Antibiot 1986;39:761-71.

49. Selan L, Berlutti F, Passariello C, Comodi-Ballanti MR, Thaller MC. Proteolytic enzymes: A new treatment strategy for prosthetic infections? Antimicrob Agents Chemother 1993;37:2618-21.

50. Aratani H, Tateishi H, Negita S. Studies on the distributions of antibiotics in the oral tissues: Experimental staphylococcal infection in rats, and effect of Serration peptidase on the distributions of antibiotics (author's trans1). Jpn J Antibiot 1980;33:623-35.

51. Ishihara Y, Kitamura S, Takaku F. Experimental studies on distribution of cefotiam, a new beta-lactam antibiotic, in the lung and trachea of rabbits. II. Combined effects with Serration peptidase. Jpn J Antibiot 1983;36:2665-70.

52. Neubauer RA. A plant protease for potentiation of and possible replacement of antibiotics. Exp Med Surg 1961;19:143.

53. Tachibana M, Mizukoshi O, Harada Y, Kawamoto K, Nakai Y. A multicentre, double-blind study of serrapeptase versus placebo in postantrotomy buccal swelling. Pharmatherapeutica 1984;3:526-30.

54. Garg R, Aslam S, Garg A, Walia R. A prospective comparative study of Serration peptidase and aceclofenac in upper and lower limb soft tissue trauma cases. Int J Pharmacol Pharm Technol 2012;1:11.

55. UmaMaheswari T, Hemalatha $T$, Sankaranarayanan $P$, Puvanakrishnan R. Enzyme therapy: Current perspectives. Indian J Exp Biol 2016;54:7-16.

56. Shahid S. Role of systemic enzymes in infections. Webmed Central Complement Med 2012;3:WMC002504

57. Maurya P, Bajpai M. Study of secnidazole - Serratio peptidase alginate/HPMC gels for periodontal delivery. Int $\mathrm{J}$ PharmTech Res 2011;3:1488-94.

58. Shah SA, Nerurkar RP. Evaluation of prescribing trends and rationality of use of oral proteolytic enzymes. Indian J Pharmacol 2013;45:309-10.

59. Kang TS, Stevens RC. Structural aspects of therapeutic enzymes to treat metabolic disorders. Hum Mutat 2009;30:1591-610.

60. Bannenberg GL. Resolvins: Current understanding and future potential in the control of inflammation. Curr Opin Drug Discov Devel 2009; $12: 644-58$ 